\title{
Evaluation of an Off-Screen Visualization for Magic Lens and Dynamic Peephole Interfaces
}

\author{
Niels Henze \\ University of Oldenburg \\ Oldenburg, Germany \\ niels.henze@uni-oldenburg.de
}

\author{
Susanne Boll \\ University of Oldenburg \\ Oldenburg, Germany \\ susanne.boll@uni-oldenburg.de
}

\begin{abstract}
Map navigation is often limited due to the inherent size restrictions of mobile devices' displays. Using a magic lens to interact with physical objects has been proposed as a way to reduce this limitation. The dynamic peephole interface is an alternative approach where a device is moved across a virtual surface. In this paper we study the effect of an additional visualization of objects beyond the screen on magic lens and dynamic peephole interfaces. In the conducted experiment the participants had to select points of interest shown on a map. We show that an additional visualization of off-screen objects decreases the task completion time and reduces the perceived task load. The advantage of an off-screen visualization is much higher than the difference between using a magic lens instead of a dynamic peephole interface.
\end{abstract}

\section{Categories and Subject Descriptors}

H.5.1 [Multimedia Information Systems]: Artificial, augmented, and virtual realities

\section{General Terms}

Human Factors, Experimentation

\section{Keywords}

Magic lens, dynamic peephole, mobile interaction, augmented reality, map navigation

\section{INTRODUCTION}

Map navigation with handheld devices helps mobile users to understand and explore their current location. However, interaction with digital maps is limited by the device's inherent small screen size. It is often difficult to identify and comprehend the distribution and position of landmarks using maps shown on mobile handsets. Traditional scrolling and panning interfaces with joystick or touch screen input offer only limited support in exploring large-scale maps on those small displays. Paper maps and public maps are often found in the city centre to provide an overview about an area. However, paper maps only contain generic information as such and places of general interest. More specific information, such as the locations of ATMs, shops, and restaurants

Copyright is held by the author/owner(s).

MobileHCI'10, September 7-10, 2010, Lisbon, Portugal.

ACM 978-1-60558-835-3/10/09. as well as short-lived events are omitted because of the limited space and the static nature of paper maps.

An approach to combine the visualization of detailed and personalized information provided by digital maps with the provision of an overview is the so-called Magic Lens $[2,10]$ used in conjunction with a paper map. The idea behind the Magic Lens is to provide an overview through the physical surface while personalized information is displayed on the phone's screen. A Magic Lens for mobile handsets [9] uses a mobile phone's display as an augmented reality window. The video stream from the phone's camera is enriched with additional information that augments physical surfaces. By determine the phone's position in relation to a static paper map, dynamic information can be merged with the camera video that is presented on the phone's screen. The Magic Lens is used to augment static paper maps with a higher level of detail, personalized information, or shortlived events. An example is using a Magic Lens to provide tourists with up-to-date information about nearby events, ratings of restaurants, and routes.

A concurrent approach that relies on the mobile phone's physical position and movement is using the device as a dynamic peephole (hereinafter referred to as peephole) $[12,5]$ that serves as a window into a virtual space. Similar to the Magic Lens the user moves the handheld device and the visualization is updated according to the device's position. In contrast to a Magic Lens, information is not visualized relative to a physical surface but relative to a virtual surface. Thus, the Magic Lens and the peephole interface provide the same visualization on the device's screen. The difference is that the Magic Lens provides additional visual context through the underlying physical surface while the peephole interface only visualizes information on the mobile device's screen itself.

Both techniques have been used to show POIs on maps (e.g. $[8,6])$. In this case, the difference between the peephole and the Magic Lens is that the paper map used in conjunction with the Magic Lens enables users to locate POIs on the map that are currently not visible on the mobile device's screen. Conceptually the paper map is used to visualize the "off-screen objects". In this paper we compare the off-screen visualization provided by the Magic Lens with off-screen visualizations developed for maps shown on mobile devices. Visualization techniques for off-screen objects are applied to a Magic Lens and a peephole interface. We show that using an arrow-based visualization for off-screen objects in combination with a Magic Lens (as shown in Figure 1) or in 


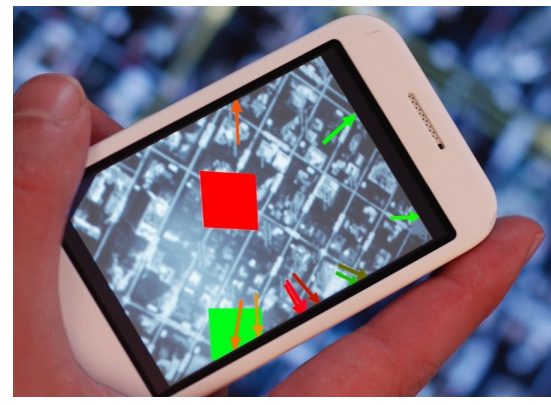

Figure 1: Map augmented with colored objects and arrows pointing at object beyond the display.

combination with a peephole interface lowers the task completion time and decreases the perceived task load.

\section{RELATED WORK}

The Magic Lens concept was introduced as a tool to filter $2 \mathrm{D}$ visualizations [2]. The concept has been adapted for mobile devices [9]. Physical objects, such as a paper map, serve as a frame of reference. A mobile device showing the device's camera image is used as the Magic Lens. When moved across the map personalized information is dynamically embedded in the camera image. Morrison et al. compared a Magic Lens for a paper map with a digital map [6]. Whereas Magic Lens interfaces are based on the idea of dynamic augmentation of real world scenes, with a dynamic peephole interface $[12,5]$ the viewport of a mobile device is used as a window into a virtual space. No visual context outside the device display is provided. Werkhoven et al. showed that a dynamic peephole interface has certain advantages compared to static peephole interfaces (i.e. interfaces were the user has to scroll the content instead of moving the device) [11]. Rohs et al. compared user's performance in a find-and-select task using a joystick controlled "static peephole", a dynamic peephole and a Magic Lens interface [8]. The study showed that switching attention between the surface and background affects task performance, yet peephole and Magic Lens clearly outperform joystick navigation. Rohs et al. also studied the impact of item density on the utility of visual context in Magic Lens interactions [7]. They found that the effectiveness of the Magic Lens compared to the peephole interface depends on the density of the virtual items. Compared to a peephole interface the Magic Lens was most effective for sparsely distributed items.

The physical map used in conjunction with Magic Lenses is a way to convey information about items beyond the spatial area covered by the device's display. Conveying information about so-called off-screen objects for digital maps has first been studied by Baudisch and Rosenholtz [1]. They developed Halo a visualization technique based on circles around the objects that intersect the display's border. Burigat et al. [3] and our own work [4] showed that arrows encoding the distance and the directions of off-screen objects are more effective than the Halo approach. A Magic Lens can be seen as an extension to the peephole interface that provides an additional off-screen visualization. So far, no study compared this off-screen visualization with other visualization techniques for off-screen objects. In particular, it hasn't been studied how user performance changes if an additional off-screen visualization is applied to a Magic Lens or a dynamic peephole interface.

\section{USER STUDY}

The experiment investigates the effect of visualizing offscreen objects using a Magic Lens and a peephole interface. To make the experiment comparable with previous studies we use a design similar to [8]. The participants had to select POIs on a map. The independent variables are the interaction technique (Magic Lens or peephole) and the visualization technique (off-screen visualization or no off-screen visualization). The dependent variables are the tasks completion time, error rate, and the perceived task load measured using the NASA TLX.

Our hypothesis was that the off-screen visualization reduces the task completion time for the Magic Lens and the peephole. This hypothesis is based on the assumption that the off-screen visualization provides additional cues but does not interfere with the augmentation. However, we assumed that interpreting the visualization of off-screen objects increases the perceived task load in particular the mental demand. Regarding the differences between the Magic Lens and the peephole we expected only small effects that are consistent with the results reported by Rohs et al. [8].

\subsection{Interaction Design and Setup}

In order to study the effect of using an off-screen visualization in combination with a Magic Lens or a peephole interface we implemented both interaction techniques. A map which is displayed on a large screen is used as static surface. We used a display instead of a paper map to be able to exchange the map. In order to make the conditions as similar as possible the screen shows a map in all conditions. I.e. the map itself was always visible but the positions of the POIs were only visible if the Magic Lens was used. For the peephole interaction the screen showed a bare map without the POIs. The map was shown for the peephole condition to eliminate effects on the user caused by different backgrounds.

The location of the POIs was chosen randomly. For the Magic Lens the POIs are marked with blue rectangles on the underlying map. A mobile phone was used as a Magic Lens and as the peephole. The phone displays the video from its camera. If the phone's camera is pointing at the map an augmentation is embedded in the video. The position of the POIs is marked with colored rectangles on the phones screen. Stretched arrows are used to visualize POIs which are currently not visible in the camera image. The arrow's length is scaled according to the distance of the respective POI from the edge of the phone's screen. Each arrow has the same color as the respective POI. Figure 2 shows the visualization with arrows pointing towards off-screen objects. In this example the object's position is also displayed on the underlying map.

We implemented the prototype using a 30" Apple Cinema Display to display the underlying map. An HTC G1 phone was used as Magic Lens and as peephole. To estimate the pose of the phone in relation to the map the video from the phone is transmitted to a server via WiFi. The server analyses the video and estimates the phone's pose which is transmitted back to the phone. Image processing is performed at a rate of around $8 \mathrm{~Hz}$. To select a POI the user 


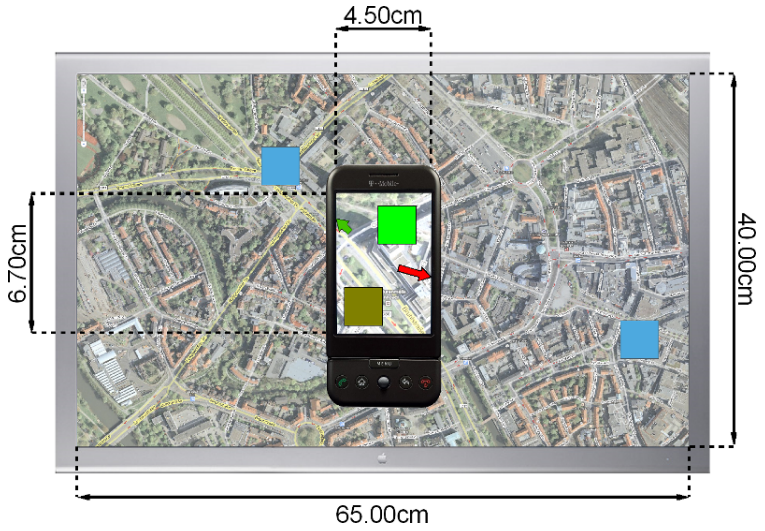

Figure 2: Conception of the visualization and hardware specification.

has to tap it with the finder on the phone's screen. Once selected, the POI fades to grey.

\subsection{Experimental Design and Procedure}

The study consisted of two tasks: In the find-and-select task participants were asked to select the "greenest" POI displayed by the augmentation on the phone. The task consisted of six sub-tasks. The participant had to select the greenest POI out of $2,4,6,8,10$, and 12 POIs. The POIs' colors were selected from a color space spanning from green to red. The order of the sub-tasks was randomized. In the select-in-order task participants were asked to select all POIs from the greenest to the reddest. There were sets with 2, 4, and 6 POIs resulting in three sub-tasks. The POIs' colors were also selected from a color space spanning from green to red.

Both tasks were set up as a $2 \times 2$ repeated measurement design with the following factors: Interaction technique: Magic Lens or peephole. Off-screen visualization: Visualization of off-screen POIs or no visualization of off-screen POIs. Participant performed both tasks with every condition. The order of the conditions was counterbalanced to minimize sequence effect. 12 persons ( 4 female) participated in the study. Most participants were students aged between 22 and 38 years (mean age 30.42). For each sub-task the taskcompletion time was measured. In addition, it was recorded if a wrong POI was selected. To assess the participants' perceived task load we used questionnaires with the unweighted version of the NASA TLX.

Participants were first familiarized with the procedure, the set up of the study, and the NASA TLX questionnaire. Before each task a textual description of the task was given on the mobile phone's screen. By tapping the screen the participant started to perform the respective sub-tasks. Participants were not asked to provide feedback while performing the tasks. After finishing one task with one condition the participant filled the questionnaire. After finishing all tasks participants were debriefed.

\section{RESULTS}

In the following we report the experiment's results. Due to size restrictions we only report significant results. In total, participants made 19 errors in the first task and 13 errors in the second task but we did not find significant differences.
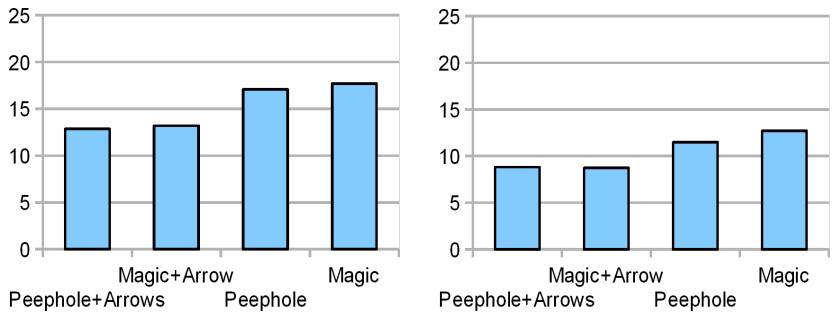

Figure 3: Task completion time for the first task (left) and the second task (right).

\subsection{Completion time}

Figure 4.1 (left) shows the average completion times for the find-and-select tasks. Completion time for the peephole with off-screen visualization was significantly faster than for the Magic Lens without off-screen visualization $(p<0.01$, $\mathrm{r}=0.47)$ and the peephole without off-screen visualization $(\mathrm{p}<0.01, \mathrm{r}=0.46)$. Completion time for the Magic Lens with off-screen visualization was also significantly faster than for the Magic Lens without off-screen visualization $(p<0.01$, $\mathrm{r}=0.51$ ) and the peephole without off-screen visualization $(\mathrm{p}<0.01, \mathrm{r}=0.41)$.

Figure 4.1 (right) shows the average time to select an item for the select-in-order tasks. Selection time for the peephole with off-screen visualization was significantly shorter than for the Magic Lens without off-screen visualization $(\mathrm{p}<0.05$, $\mathrm{r}=0.10)$ and the peephole without off-screen visualization $(\mathrm{p}<0.01, \mathrm{r}=0.22)$. Selection time for the Magic Lens with off-screen visualization was also significantly shorter than for the Magic Lens without off-screen visualization $(\mathrm{p}<0.01$, $\mathrm{r}=0.12$ ) and the peephole without off-screen visualization $(\mathrm{p}<0.001, \mathrm{r}=0.23)$.

\subsection{Subjective Results}

Analysis of the questionnaires showed that conditions with additional off-screen visualization were rated lower demanding for both tasks. The unweighted NASA TLX score for the peephole with off-screen visualization $(\varnothing=4.53)$ was significantly lower than for the Magic Lens without off-screen visualization $(\varnothing=5.74, \mathrm{p}<0.01, \mathrm{r}=0.77)$ and the peephole without off-screen visualization $(\varnothing=6.49, \mathrm{p}<0.01, \mathrm{r}=0.71)$. The unweighted NASA TLX score for the Magic Lens with off-screen visualization $(\varnothing=5.12)$ was also significantly lower than for the Magic Lens without off-screen visualization $(\mathrm{p}<0.05, \mathrm{r}=0.75)$ and the peephole without off-screen visualization $(\mathrm{p}<0.05, \mathrm{r}=0.66)$. We found a number of significant differences for the individual NASA TLX values that are all consistent with the result for the unweighted TLX score. A mentionable significant result $(\mathrm{p}<0.05, \mathrm{r}=0.68)$ is that the peephole with off-screen visualization was rated less mentally demanding $(\varnothing=5.42)$ than the Magic Lens with offscreen visualization $(\varnothing=7.25)$.

The unweighted NASA TLX score for the peephole with off-screen visualization $(\varnothing=5.36)$ was significantly lower than for the Magic Lens without off-screen visualization $(\varnothing=7.04$, $\mathrm{p}<0.01, \mathrm{r}=0.79)$ and the peephole without off-screen visualization $(\varnothing=7.47, \mathrm{p}<0.01, \mathrm{r}=0.83)$. The unweighted NASA TLX score for the Magic Lens with off-screen visualization $(\varnothing=4.82)$ was also significantly lower than for the Magic Lens without off-screen visualization $(\mathrm{p}<0.01, \mathrm{r}=0.75)$ and the peephole without off-screen visualization $(\mathrm{p}<0.001, \mathrm{r}=0.88)$. 
We found a number of significant differences for the individual NASA TLX values that are all consistent with the result for the unweighted score. A mentionable significant result $(\mathrm{p}<0.05, \mathrm{r}=0.66)$ is that the peephole with off-screen visualization was rated more mentally demanding $(\varnothing=7.17)$ than the Magic Lens with off-screen visualization $(\varnothing=5.58)$. Furthermore, the Magic Lens without off-screen visualization was rated less mentally demanding $(\varnothing=7.67)$ than the peephole without off-screen visualization $((\varnothing=9.08, \mathrm{p}<0.05$, $\mathrm{r}=0.53)$.

\section{DISCUSSION}

The experiment supports our hypothesis that visualization of off-screen objects reduces the task completion time. Visualization of off-screen objects lead to a task completion time that is about one quarter lower. Furthermore, we also observed the time difference between Magic Lens and peephole is much smaller than the difference between off-screen visualization and no off-screen visualization. Unlike [7] we did not find significant differences between the peephole and the Magic Lens. Based on our data we assume that our third hypothesis, that differences between the Magic Lens and the peephole interface are negligible for the performed tasks and setup, is also valid.

Contrary to our second hypothesis the perceived task load is lower if off-screen objects are visualized. Even though participants had to interpret the additional visualization it seems that the arrows assisted the participants more than we expected. In addition, the off-screen visualization might have reduced visual context switches between the phone's display and the physical map. Differences between the peephole and the Magic Lens regarding mental demand were not consistent for the two tasks. Since participants rated the Magic Lens less mentally demanding in the second task we assume that training might have a larger effect for the Magic Lens than for the peephole interface.

Overall we observed the same trend for the ratings by the participants and the measured task completion time. If off-screen visualizations are used participants were more efficient and they perceived the interface as more efficient. We assume that for tasks as those used in this study it is questionable if the Magic Lens provides any relevant benefit over the peephole interface. We can, however, not predict the effect of off-screen visualization in more complex tasks. If larger surfaces are used and the density of off-screen objects is increased the arrows used in this study will start to overlap which will clearly reduce the effectiveness of this particular visualization at a given point.

\section{CONCLUSIONS AND FUTURE WORK}

In this paper we showed that an off-screen visualization for Magic Lens and peephole interfaces improves users' performance. Participants gain a considerably larger benefit from a visualization of off-screen objects than from using a Magic Lens instead of a peephole interface. It has been shown (e.g. $[8,7]$ ) that a paper map used in conjunction with a Magic Lens provides a significant benefit. Our results, however, suggest that this benefit is negligible if an off-screen visualization is used - at least for simple tasks.

Even though in our study the user's interaction range was restricted by the physical object, peephole interfaces can also be designed with a user defined frame of reference. Consid- ering that no benefit for a Magic Lens has been found for our tasks we suggest that one should be careful to expect a significant benefit for other tasks as well. Using physical objects as an anchor to select digital content might be beneficial. However, using traditional "static-peephole" interfaces or a peephole interface with off-screen visualization could be the superior interaction technique after selecting the content.

The simple tasks used in the study did not require the user to examine the map in detail or derive spatial decisions. Future research should investigate the benefit of a Magic Lens with off-screen visualization for users performing complex tasks. In particular, tasks that require survey knowledge and involve multiple users. Furthermore, most research regarding handheld augmented reality focused on interaction with planar objects. We plan to investigate if our findings can be applied to interaction with three-dimensional objects.

\section{ACKNOWLEDGEMENT}

This paper is supported by the European Community within the InterMedia project (project No. 038419).

\section{REFERENCES}

[1] P. Baudisch and R. Rosenholtz. Halo: a technique for visualizing off-screen objects. In Proc. of CHI, 2003.

[2] E. Bier, M. Stone, K. Pier, W. Buxton, and T. DeRose. Toolglass and Magic Lenses: The See-Through Interface. In Proc. of SIGGRAPH, 1993.

[3] S. Burigat, L. Chittaro, and S. Gabrielli. Visualizing locations of off-screen objects on mobile devices: a comparative evaluation of three approaches. In Proc. of MobileHCI, 2006.

[4] N. Henze and S. Boll. Push the study to the app store: Evaluating off-screen visualizations for maps in the android market. In Adjunct Proc. of MobileHCI, 2010.

[5] S. Mehra, P. Werkhoven, and M. Worring. Navigating on handheld displays: Dynamic versus static peephole navigation. TOCHI, 13(4):448-457, 2006.

[6] A. Morrison, A. Oulasvirta, P. Peltonen, S. Lemmela, G. Jacucci, G. Reitmayr, J. Näsänen, and A. Juustila. Like bees around the hive: a comparative study of a mobile augmented reality map. In Proc. of CHI, 2009.

[7] M. Rohs, R. Schleicher, J. Schöning, G. Essl, A. Naumann, and A. Krüger. Impact of item density on the utility of visual context in magic lens interactions. Personal and Ubiquitous Computing, pages 1-14, 2006.

[8] M. Rohs, J. Schöning, M. Raubal, G. Essl, and A. Krüger. Map navigation with mobile devices: virtual versus physical movement with and without visual context. In Proc. of ICMI, 2007.

[9] J. Schöning, A. Krüger, and H. J. Müller. Interaction of mobile camera devices with physical maps. Adjunct Proc. of Pervasive, 2006.

[10] J. Viega, M. Conway, G. Williams, and R. Pausch. 3D magic lenses. In Proc. of UIST, 1996.

[11] P. Werkhoven and M. Worring. A Proof of Concept for Spatially Tracked Hand-Held Displays. In Proc. of EUROCON, 2005.

[12] K.-P. Yee. Peephole displays: pen interaction on spatially aware handheld computers. In Proc. of $\mathrm{CHI}$, 2003. 